\title{
Results of Reconstructive Surgery in the Upper Limb of Tetraplegic Patients
}

\author{
A. Ejeskär, ${ }^{1}$ A. Dahllöf. ${ }^{2}$ \\ ${ }^{1}$ Department of Orthopaedic Surgery, Department of Rehabilitation Medicine, \\ Sahlgren Hospital, Goteborg, Sweden
}

\begin{abstract}
Summary
Forty three out of 62 tetraplegic patients operated on in Sweden during 1970 to 1984 with various reconstructive procedures in the arm and hand have been reexamined. The results show that reconstruction of elbow extensor, key grip and finger flexor are all very valuable procedures if performed on correct indications. All but 4 patients improved by surgery and in many instances the surgical treatment facilitated return to work.
\end{abstract}

Key words: Tetraplegia; Reconstructive surgery; Elbow extensor, Key grip; Finger flexor.

Up to 15 to 20 years ago the general opinion was that function in the arm and hand of tetraplegic patients could not be improved by surgery. However, at the end of the 1960s the first positive reports on successful operative treatment appeared and in 1970 Moberg started his pioneering work, classifying these patients from a functional point of view and treating some of them operatively.

\section{Patients and methods}

\section{Classification}

During the years 1970 to 1984 Moberg treated 65 patients living in Sweden of which 62 still are alive, living in various parts of the country. Up to the time of acceptance of this paper 43 of these 62 patients have been re-examined. The preoperative classification of the best arm of these patients according to the system of Moberg (1978) described is shown in Table I.

Eleven patients lacked useful tactile gnosis in both hands and two thirds (28/43) had a maximum of two muscles which were of grade 4 or 5 thus belonging to the groups $0: 1,0: 2,0 \mathrm{Cu}: 1$ or $0 \mathrm{Cu}: 2$. Only 28 of the patients had symmetrical function in both arms.

\section{Operative techniques}

The operative treatment included a variety of procedures but in this report we will limit ourselves to the results of the three most frequent operations, namely 
Table I Preoperative classification of the best arm in 43 reexamined patients

\begin{tabular}{cc}
\hline Group & Number \\
\hline $0: 1$ & 9 \\
$0: 2$ & 2 \\
OCu $: 1$ & 4 \\
OCu $: 2$ & 13 \\
OCu $: 3$ & 9 \\
OCu $: 4$ & 5 \\
OCu $: 6$ & 1 \\
\hline
\end{tabular}

reconstruction of elbow extensor, production of the so called 'key grip', and procedures for finger flexion.

a. Elbow extensor has been reconstructed using the posterior part of the deltoid as a motor. Two different methods have been used to bridge the gap between the deltoid insertion and the triceps tendon namely (1) free tendon grafts (Moberg, 1978) and (2) reflexion of the central third of the triceps tendon according to Castro-Sierra \& Lopez-Pita (1983).

b. The key grip is based on active dorsiflexion of the wrist. The procedure originally included tenodesis of the flexor pollicis longus against the radius, temporary arthrodesis of the interphalangeal joint of the thumb and opening of the annular ligament volar to the metacarpophalangeal (MCP) joint of the thumb (Fig. 1). More recently the transpalmar key grip (Moberg 1987) has been used (Fig. 2) where the flexor pollicis longus is rerouted dorsal to the finger flexors and to the Guyon canal before it is anchored into the radius. The annular ligament volar to the MCP joint is not incised.

c. Finger flexor has been reconstructed by transferral of the extensor carpi radialis longus to the profundi $\mathrm{II}-\mathrm{V}$.

\section{Follow-up study}

The 43 patients have been re-examined 1 to 14 years after the last operation with respect to the effect of surgery. There were 7 women and $36 \mathrm{men}$, born between the years of 1918 up to 1962 .

At the follow-up examination the patients were questioned concerning their ability to perform 55 different activities of daily living (ADL) tasks, as well as their opinion of the effect of the operative treatment upon their capacity to perform these tasks. Elbow extension was evaluated with respect to any extension deficit when extending against gravity and whenever possible also holding a sandbag in the hand. The key grip pinch was measured with the help of a Preston Pinch gauge (JA Preston Corp, 60 Page Road, Clifton NJ 07012, USA), and finger flexion was evaluated by measuring the whole hand grip by means of a Martin Vigorimeter (Gebrüder Martin, Tuttlingen, Germany). The presence of contractures was noted as well as the motion in the joints of the thumb and fingers. 


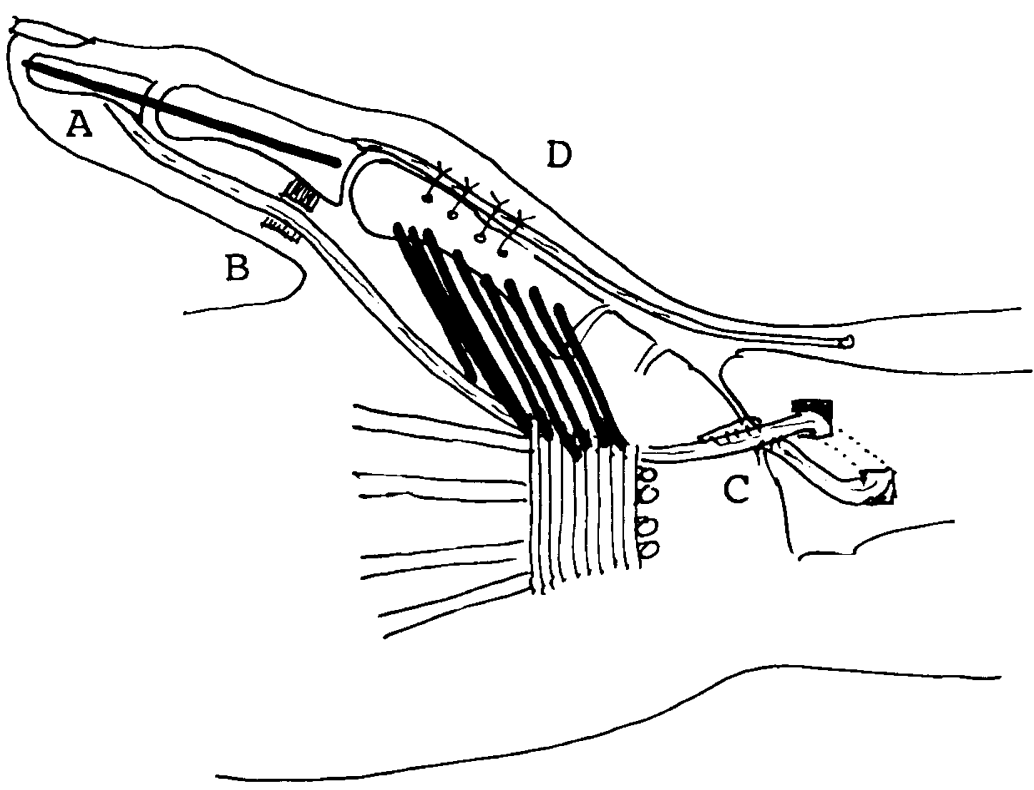

Figure 1 Key grip (original method). (A) Temporary arthrodesis of the interphalangeal joint. (B) Opening of the anular ligament volar to the MCP joint. (C) Tenodesis of the flexor pollicis longus tendon against radius. (D) Tenodesis of the extensor pollicis brevis against the first metacarpal in cases with passive flexion of the MCP joint exceeding $45^{\circ}$.

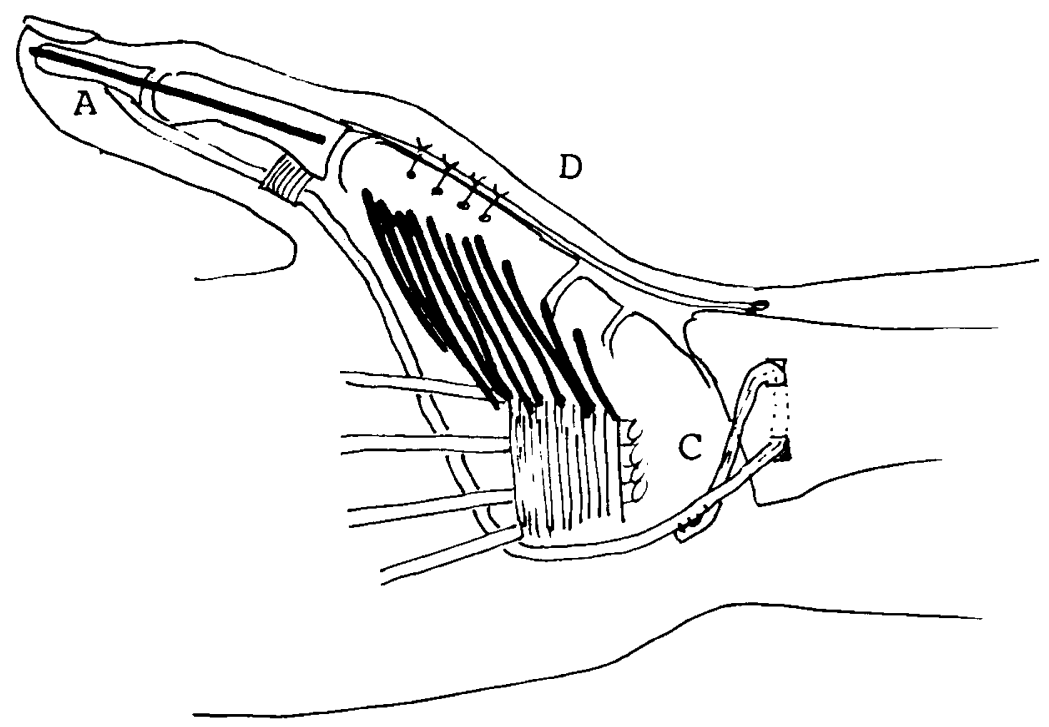

Figure 2 Transpalmar key grip. (A) Temporary arthrodesis of the interphalangeal joint. (C) Tenodesis of the flexor pollicis longus rerouted dorsal to the finger flexor tendons. (D) Tenodesis of the extensor pollicis brevis (elective, in cases with passive flexion of the MCP joint exceeding $\left.45^{\circ}\right)$. 
Table II Results of reconstruction of the elbow extensor

\begin{tabular}{lcccc}
\hline Method extension deficit & $0^{\circ}$ & $\leqslant 60^{\circ}$ & $>60^{\circ}$ & Total \\
\hline Toe extensor tendons & 5 & 8 & 10 & 23 \\
Castro-Sierra Lopez-Pita & 1 & 0 & 6 & 7 \\
Total & 6 & 8 & 16 & 30 \\
\hline
\end{tabular}

\section{Results}

a. Elbow extensors: thirty elbows in 23 patients have been examined. Twenty three of these 30 were operated on using the method with free tendon grafts, and seven with the method of Castro-Sierra \& Lopez-Pita. The results show that 5/23 elbows operated using method 1 had full extension, 8 had lack of extension against gravity of maximum $60^{\circ}$, and 10 had an even greater lack of extension (Table II). The results after operation according to Castro-Sierra \& Lopez-Pita were inferior to these, as 6 out of 7 elbows had an extension deficit greater than $60^{\circ}$.

In spite of the fact that many patients had poor elbow extension they still benefitted from the operation by a better muscular balance in the elbow, facilitating activities such as car driving and handwriting. A weak elbow extensor when tested against gravity may have sufficient strength to counteract the function of the brachioradialis, which then may be used for transfer.

b. The key grip: Fifty hands out of a total of 40 patients were operated on with this method. The strength varied from zero to $3.5 \mathrm{~kg}$ with an average of 0.7 $\mathrm{kg}$. The strength is dependant upon the power of dorsiflexion of the wrist, which in all 15 cases with a minimum of $1.0 \mathrm{~kg}$ pinch grip was judged to be at least grade 4 .

The usefulness of the grip was influenced not only by the power but also by the position of the thumb and index finger. A weak key grip in an ideal position can be better than a strong one in a less good position. The transpalmar technique seems to induce less strong flexion in the metacarpophalangeal joint of the thumb but does not eliminate the need for a dorsal tenodesis of the extensor pollicis brevis to the first metacarpal. Flexion of more than $30^{\circ}$ in the MCP joint reduces the efficiency of the key grip by allowing the thumb to rotate in the carpometacarpal joint and by reducing the tension in the flexor pollicis longus tenodesis.

The results also showed that the position of the index finger was very important. Too much flexion at the MCP joint, that is $60^{\circ}$ or more gives less good function as the thumb will have a tendency to be 'too long' compared to the finger. When splinting the hands in tetraplegic patients we therefore recommend that the MCP joints should not be flexed more than $45-50^{\circ}$.

c. Finger flexion: Fourteen hands in 13 patients were operated on with transfer of the ECRL to the profundi II-V. The strength of the whole hand grip ranged from zero to $0 \cdot 27 \mathrm{kP}$ with an average of $0 \cdot 13$. Five out of these 14 patients had a key grip strength of minimum $1.0 \mathrm{~kg}$.

Subjectively 4 patients said that they were not improved by the treatment. One had severe spasticity which nowadays would have been considered to be a definite contraindication to surgery. In 2 of these 4 patients the brachioradialis 
muscle had been transferred to reinforce dorsiflexion of the wrist in the absence of an elbow extensor. The effect of the transfer was thereby minimal. The fourth patient was a man who at his own request had an operation on his weaker hand and never benefitted from the operation. Four patients were unable to state how much they had improved by the treatment while the remaining 35 patients noted an average improved capacity to perform 23 out of 55 ADL tasks.

Three of the 43 patients had experienced a functional detriation the last one and a half years preceding the follow-up examination. However, this could not be attributed to the treatment but possibly to the existence of peripheral nerve compressions.

Twenty seven of the patients were working, in must instances part time, and another 3 patients were studying, hoping to obtain some type of work after completing their studies. In many instances the surgical treatment had considerably facilitated their return to work.

Contractures in the interphalangeal joints of the fingers exceeding a total of $60^{\circ}$ was noted in 2 patients; in 1, a finger flexor had been reconstructed without the existence of a finger extensor. However, the contractures had pre-existed the operation. In other patients, only a key grip procedure had been performed. In no patient had a transfer of the extensor carpi radialis longus to the profundi with its 'imbalance' of the fingers created the contracture.

Thus where no doubt that surgery can improve the function in the arm and hand of most tetraplegic patients if the indications are correct, and if the patient is well motivated. The surgeon should then always firstly operate on the best arm.

It is also quite clear that the operative methods may be further improved, and our study clearly reveals the great need for a regular follow-up of these patients from a hand/surgical point of view.

\section{Acknowledgment}

The study was supported by the Greta and Einar Asker's foundation.

\section{References}

CASTRO-Sierra A, Lopez-Pita A 1983 A new surgical technique to correct triceps paralysis. The Hand 15: 42.

MoBERG ERIK 1978 The Upper Limb in Tetraplegia. G. Thieme, Stuttgart.

MOBERG ERIK 1987 Current treatment program using tendon surgery in Tetraplegia. In Tendon Surgery in the hand Ed. Hunter, J, Schneider L. and Mackin E. C.V. Mosby, St Louis, Washington DC pp 496-505. 